\title{
Influence of renal denervation on blood pressure, sodium and water excretion in acute total obstructive apnea in rats
}

\author{
J.V.M. Franquini², A.R.S. Medeiros ${ }^{1}$, T.U. Andrade ${ }^{1,2}$, M.T.M. Araújo ${ }^{1}$, M.R. Moysés ${ }^{1}$, \\ G.R. Abreu ${ }^{1}$, E.C. Vasquez ${ }^{1}$ and N.S. Bissoli ${ }^{1}$ \\ 1'Departamento de Ciências Fisiológicas, Centro de Ciências da Saúde, Universidade Federal do Espírito \\ Santo, Vitória, ES, Brasil \\ 2Departamento de Farmácia, Universidade de Vila Velha, Vila Velha, ES, Brasil \\ Correspondence to: N.S. Bissoli, Departamento de Ciências Fisiológicas, Centro de Ciências da Saúde, \\ Universidade Federal do Espírito Santo, Av. Marechal Campos, 1468, 29040-090 Vitória, ES, Brasil \\ Fax: +55-27-3335-7340. E-mail: naza@npd.ufes.br
}

\begin{abstract}
Obstructive apnea (OA) can exert significant effects on renal sympathetic nerve activity (RSNA) and hemodynamic parameters. The present study focuses on the modulatory actions of RSNA on OA-induced sodium and water retention. The experiments were performed in renal-denervated rats $(D ; N=9)$, which were compared to sham $(S ; N=9)$ rats. Mean arterial pressure $(M A P)$ and heart rate $(\mathrm{HR})$ were assessed via an intrafemoral catheter. A catheter was inserted into the bladder for urinary measurements. OA episodes were induced via occlusion of the catheter inserted into the trachea. After an equilibration period, OA was induced for 20 s every 2 min and the changes in urine, MAP, HR and RSNA were recorded. Renal denervation did not alter resting MAP (S: $113 \pm 4$ vs D: $115 \pm 4 \mathrm{mmHg}$ ) or HR (S: $340 \pm 12$ vs D: $368 \pm 11 \mathrm{bpm}$ ). An OA episode resulted in decreased $\mathrm{HR}$ and MAP in both groups, but $\mathrm{D}$ rats showed exacerbated hypotension and attenuated bradycardia $(\mathrm{S}:-12 \pm 1 \mathrm{mmHg}$ and $-16 \pm 2$ bpm vs D: $-16 \pm 1 \mathrm{mmHg}$ and $9 \pm 2 \mathrm{bpm} ; \mathrm{P}<0.01$ ). The basal urinary parameters did not change during or after $\mathrm{OA}$ in $S$ rats. However, $D$ rats showed significant increases both during and after OA. Renal sympathetic nerve activity in $S$ rats increased (34 $\pm 9 \%)$ during apnea episodes. These results indicate that renal denervation induces elevations of sodium content and urine volume and alters bradycardia and hypotension patterns during total $O A$ in unconscious rats.
\end{abstract}

Key words: Obstructive apnea; Renal nerve; Natriuresis; Diuresis

Received July 9, 2008. Accepted January 26, 2009

\section{Introduction}

Obstructive apnea (OA) events during sleep affect normal respiratory and cardiovascular functions $(1,2)$. It is well established that apnea, especially OA, can exert a significant effect on renal sympathetic nerve activity (RSNA) $(1,3)$, which is well known to play a pivotal role in cardiovascular reflexes, including the chemoreflex. This reflex may be activated when airway obstruction produces hypoxemia with oxyhemoglobin desaturation and a significant increase in $\mathrm{PaCO}_{2}(3,4)$. The responses induced by chemoreflex activation are a rise in sympathetic nerve activity to the kidneys and other vascular beds, as well as an increase in the activity of vagal efferents on heart rate
(HR) (5). In addition to the chemoreflex, other cardiovascular reflexes such as cardiopulmonary reflexes, and vasoactive hormones could be involved simultaneously in modifications of the kidney's excretory responses to OA, which include a reduced RSNA (6-11).

Although systemic hypoxia has pronounced effects on renal function through RSNA and/or vasoactive hormones, the nature of these effects is still unclear. For example, it has been reported that systemic hypoxia induces antidiuresis and antinatriuresis in anesthetized dogs (12) and fetal lambs (13), conscious rats (14) and rabbits (15), and human subjects (16). In contrast, diuresis and natriuresis have been reported in conscious dogs $(17,18)$, in human subjects $(19,20)$ and in anesthetized rats $(21)$. The wide 
variety of responses obtained in these studies can be attributed to differences in animal species, degree of hypoxia and state of anesthesia.

Therefore, the goal of the present study was to investigate the influence of RSNA on sodium and water excretion, and hemodynamic parameters during acute severe OA episodes in anesthetized rats, caused by ineffective inspiratory efforts against total occlusion of the upper airways.

\section{Material and Methods}

Experiments were performed on male Wistar rats (240$260 \mathrm{~g}$ ) obtained from the animal care facilities of the Physiological Sciences Graduate Program of the University Federal of Espírito Santo. All procedures were conducted in accordance with the biomedical research guidelines for the care and use of laboratory animals as stated by the Federation of the Brazilian Societies of Experimental Biology (FeSBE). Rats were housed at recommended levels of temperature and humidity in a room with a 12-h light/dark cycle. Standard rat chow ( $\mathrm{Na}^{+}$content $163 \mathrm{mEq} /$ $\mathrm{kg}$ ) and tap water were available ad libitum.

\section{Renal denervation}

Rats underwent bilateral renal denervation to eliminate the neural influence on renal excretory functions. Under sodium thiopental (50 mg/kg, ip) anesthesia, the left kidney was exposed via a flank incision. The adventitia surrounding the renal artery and vein was stripped and all visible renal nerves were cut under a surgical microscope (902DF Vasconcellos, São Paulo, SP, Brazil). The vessels were then treated with $95 \%$ alcohol containing $10 \%$ phenol. After renal denervation the flank incision was sutured and the procedure was repeated on the opposite side to denervate the right kidney. This surgical procedure was performed 15 days before the experimental protocol because it is known that it prevents the renal vasoconstrictor response to suprarenal lumbar sympathetic nerve stimulation and the antinatriuretic response to environmental stress and reduces renal tissue norepinephrine concentration to $<5 \%$ of control for up to 15 days post-denervation $(22,23)$.

After the acute experiments, the kidneys were removed under anesthesia and stored frozen until norepinephrine was measured. The success of the renal denervation procedure was confirmed by the reduction of the quantity of renal tissue norepinephrine to undetectable values in the renal-denervated (D) group compared to $382 \pm 25 \mathrm{ng} /$ $\mathrm{g}$ wet weight kidney in the sham-operated (S) group.

\section{Experimental protocol}

Fifteen days after denervation, on the day of the acute experiment, rats were anesthetized with urethane $(1 \mathrm{~g} / \mathrm{kg}$, ip) and supplemented with the same anesthetic (iv) as needed. A polyethylene catheter was placed into the femoral artery and vein to measure mean arterial pressure (MAP) and HR and for infusion of isotonic saline, respectively. The catheter was tunneled subcutaneously to the back of the neck, flushed and plugged. For urinary measurements, a suprapubic incision was made and a polyethylene catheter was inserted and sutured into the bladder. This catheter was then exteriorized and secured by suturing it to the adjacent muscle and skin (23). Rats also underwent tracheal catheterization to induce severe $O A$ episodes via total occlusion of the tracheal polyethylene catheter.

\section{Renal excretory responses}

Experiments comparing the natriuretic and diuretic responses induced by OA were performed under anesthesia. Initially, the animals received iv infusions of isotonic saline $(55 \mu \mathrm{L} / \mathrm{min})$ to enhance renal excretion of water and sodium (23). After an equilibration period for the stabilization of renal excretory responses the experimental protocol was performed and divided into 3 periods: control $(C)$, apnea $(A)$ and recovery $(R)$. During the control period, two consecutive urine samples ( $\mathrm{C} 1$ and $\mathrm{C} 2$ ) were collected (10 min each). During the apnea period, urine was collected during five intervals of $10 \mathrm{~min}$ ( $\mathrm{A} 1, \mathrm{~A} 2, \mathrm{~A} 3, \mathrm{~A} 4$, and $\mathrm{A5}$ ). During each of these periods, five OA episodes were performed (20 s each), with 2-min intervals between them. After the OA episodes, urine was collected five consecutive times (10 min each) during the recovery period (R1, R2, R3, R4, and R5).

Urinary volume and sodium content, MAP and HR were determined at all time. For saline infusion, we used an infusion pump (model 600-900V; Harvard Apparatus, USA). The arterial catheter was connected to a pressure transducer, model P23Db (Statham, USA). Throughout the experiment MAP and HR were recorded continuously using a polygraph (Sensormedics Dynograf Recorder $\mathrm{R}$ 711, USA).

\section{Nerve activity}

In a separate experimental group, rats were anesthetized with urethane $(1 \mathrm{~g} / \mathrm{kg}, i p)$ and the left kidney was exposed via a retroperitoneal approach through a left flank incision. Using a dissecting microscope (M 900 DF, Vasconcellos, São Paulo, SP, Brazil), renal nerves were identified, isolated, and carefully dissected. The renal nerve branch was then placed on a bipolar platinum wire electrode and gelled with silicone. Extracellular action potentials were recorded with an AC amplifier (NL 104, NeuroLog, 
Digitimer, England) connected to a high impedance headstage (NL 100). The amplified signals were filtered (NL 126), connected to an audio amplifier (NL 120) and displayed on an oscilloscope (Tektronix 2205, Brazil). The data were processed using a spike trigger (NL 200) and a ratemeter (NL 256) and displayed on a Biopac System (MP100). All data were digitized and stored (Digital MTE 46602 Tape Stream, USA) for further analysis (Acknowledge for Windows; Biopac Inc., USA) (24). After completion of surgical preparation and an equilibration period, RSNA was quantified by measuring the integrated RSNA during a 20-s apnea episode. MAP and HR were monitored simultaneously during the OA. We determined the background noise level of RSNA by observing the neural signal that remained after the animals were euthanized by an overdose of urethane and this value was subtracted from all control and experimental RSNA values.

\section{Examination of excretory system function}

The kidneys were removed, rinsed in physiological saline, decapsulated, blotted, and weighed for normalization of renal excretory data. Urine sodium concentration was measured by flame photometry with a model B Micronal apparatus (Brazil).

\section{Statistical analysis}

All data are reported as means \pm SEM. Data for basal MAP, HR, sodium, and volume excretion and changes in these parameters evoked by OA were subjected to twoway analysis of variance (ANOVA), followed by the post hoc Tukey test for multiple comparisons. Statistical significance was set at $\mathrm{P}<0.05$.

\section{Results}

\section{Hemodynamic parameters}

Renal denervation did not change resting values of MAP (S: $113 \pm 4$ vs D: $115 \pm 4 \mathrm{mmHg}$ ) or HR (S: $340 \pm 12$ vs D: $368 \pm 11 \mathrm{bpm})$. However, OA induced significant changes in hemodynamic parameters including bradycardia and hypotension. Episodes of 20-s OA were repeated in S and D animals and also caused hypotension followed by bradycardia during the apnea periods ( $A 1$ to $A 5)$. In the $D$ group, OA caused a stronger hypotension and minor bradycardia. The results of the first apnea period (A1) multiple comparisons).
(S: $-12 \pm 1 \mathrm{mmHg}$ and $-16 \pm 2 \mathrm{bpm}$ vs D: $-16 \pm 1 \mathrm{mmHg}$ and $9 \pm 2$ bpm; $P<0.01$ ) did not change during the other apnea events (A2 to $A 5)$ in either group.

\section{OA-induced changes in sodium and water excretion}

Basal sodium and water excretion (C1 and $\mathrm{C} 2)$ did not differ between groups. However, urinary sodium and volume excretion during the periods from $A 1$ to $A 5$ and at the beginning of the recovery phase differed between $S$ and $D$ rats (Table 1). S rats displayed the same excretory pattern during the basal phase (resting values) and during the experimental (from A1 to A5) and recovery phases (from $R 1$ to R5). However, in D rats, these levels increased during $\mathrm{A} 1$ and remained elevated through the $\mathrm{A} 5$ period and during the recovery phase (Table 1 ).

\section{Renal sympathetic nerve activity}

RSNA increased by $34 \pm 9 \%$ during the 20 -s OA episodes in $\mathrm{S}$ rats $(\mathrm{N}=7)$. After the apnea events, the RSNA values always returned to basal levels.

\section{Discussion}

In the present study, we reproduced conditions close to those observed during sleep apnea under total airway blockage via tracheal occlusion (25), even though in our experiments we observed only acute effects. We demonstrated that acute total obstructive apneic events resulted in an increase in diuresis and natriuresis in D rats. Basal values of urinary sodium excretion and urine volume were

Table 1. Sodium excretion and urinary volume in renal-denervated rats during the control ( $\mathrm{C} 1$ and $\mathrm{C} 2$ ), experimental (A1 to A5), and recovery (R1 to R5) phases.

\begin{tabular}{lccccc}
\hline \multirow{2}{*}{$\begin{array}{l}\text { Experimental } \\
\text { protocol }\end{array}$} & \multicolumn{2}{c}{ Sodium excretion $\left(\mu \mathrm{Eq} \cdot \mathrm{min}^{-1} \cdot \mathrm{g}^{-1}\right)$} & & \multicolumn{2}{c}{ Urine volume $\left(\mu \mathrm{L} \cdot \mathrm{min}^{-1} \cdot \mathrm{g}^{-1}\right)$} \\
\cline { 2 - 3 } \cline { 5 - 6 } & Sham & Renal-denervated & & Sham & Renal-denervated \\
\hline C1 & $2.1 \pm 0.5$ & $2.3 \pm 0.6$ & & $16.9 \pm 3.9$ & $17.3 \pm 3.0$ \\
C2 & $2.2 \pm 1.3$ & $2.5 \pm 1.7$ & & $19.4 \pm 5.1$ & $18.1 \pm 3.1$ \\
A1 & $4.2 \pm 1.0$ & $6.8 \pm 0.5^{*+}$ & & $24.2 \pm 5.4$ & $35.1 \pm 4.3^{*+}$ \\
A2 & $3.0 \pm 1.0$ & $5.8 \pm 1.5^{*+}$ & & $19.0 \pm 4.2$ & $27.8 \pm 3.9^{*+}$ \\
A3 & $3.4 \pm 1.0$ & $6.8 \pm 1.2^{*+}$ & & $20.7 \pm 3.4$ & $32.9 \pm 4.3^{*+}$ \\
A4 & $3.8 \pm 0.9$ & $6.4 \pm 1.0^{*+}$ & & $21.8 \pm 2.2$ & $30.8 \pm 3.3^{*+}$ \\
A5 & $3.9 \pm 1.0$ & $7.7 \pm 1.2^{*+}$ & & $22.5 \pm 2.1$ & $37.6 \pm 2.2^{*+}$ \\
R1 & $3.9 \pm 1.0$ & $7.3 \pm 1.0^{*+}$ & & $22.6 \pm 5.8$ & $36.8 \pm 6.6^{*}$ \\
R2 & $4.9 \pm 1.6$ & $6.6 \pm 1.1^{*}$ & & $23.3 \pm 5.9$ & $42.2 \pm 9.2^{*}$ \\
R3 & $4.0 \pm 1.2$ & $6.0 \pm 1.0^{*}$ & & $22.7 \pm 7.9$ & $34.5 \pm 8.3^{*}$ \\
R4 & $4.6 \pm 1.6$ & $6.6 \pm 1.2^{*}$ & & $24.8 \pm 8.1$ & $36.9 \pm 7.7^{*}$ \\
R5 & $4.6 \pm 1.5$ & $7.3 \pm 1.4^{*}$ & & $24.6 \pm 7.7$ & $39.8 \pm 7.5^{*}$ \\
\hline
\end{tabular}

Data are reported as means \pm SEM for $N=9$ animals per group. ${ }^{*} \mathrm{P}<0.05$ within group comparisons. ${ }^{+} \mathrm{P}<0.05$ vs sham rats (ANOVA followed by the post hoc Tukey test for 
not affected by OA episodes in $S$ animals, but these episodes elicited falls in HR and MAP. These hemodynamic and renal alterations occurred despite an increase in RSNA. However, in D rats, OA episodes caused a significant increase in renal parameters compared to basal values. Denervation induced a higher decrease in MAP and a smaller reduction in $\mathrm{HR}$.

In the case of OA, sympathetic activity has been reported to be regulated by the chemoreflex. O'Donnell et al. (3) showed that OA led to a significant decrease in $\mathrm{PaO}_{2}$ levels and an elevation of sympathetic activity, which was attenuated when the animals breathed $100 \%$ oxygen. As the peripheral chemoreflex is first stimulated by hypoxia (5), the airway obstruction in the rats was probably sufficient to activate the peripheral chemoreceptors and, as a consequence, to increase the RSNA $(5,15)$. This could result in reduction of urinary sodium excretion and urine volume (7). Therefore, the rise in natriuresis and diuresis in renal-denervated animals during the OA sequences could directly result from renal denervation and from the consequent reduction of the chemoreflex influence on the renal excretory parameters $(3,26)$.

We cannot exclude the possible participation of other cardiovascular reflexes in the modulation of renal excretory responses during $\mathrm{OA}$, such as cardiopulmonary reflexes. This neural reflex could be similar to the Müller maneuver, as a result of the great inspiratory effort required to overcome airway obstruction. This obstruction would result in a negative intrathoracic pressure, which could cause low pressure levels and affect the intrathoracic hemodynamics (27), increasing the central venous pressure (28). These OA-induced hemodynamic alterations could stimulate the mechanical cardiopulmonary receptors as a consequence of the enhancement of the central venous pressure, probably resulting in a reduction of RSNA (24). Therefore, a balance between the chemoreflex and cardiopulmonary reflex during $O A$ could result in the maintenance of urinary excretion at basal values, as observed in this study in $\mathrm{S}$ rats. Our data show that renal nerve denervation is a critical factor in modifying diuresis during severe OA via tracheal occlusion in anesthetized rats.

In this study, the OA periods caused decreases in MAP and HR when compared to basal values (i.e., before the airway obstruction). This is consistent with studies showing a marked decrease in MAP after OA events $(4,29)$. However, studies have also shown that hypoxia does not affect MAP $(15,30)$ or that it increases due to sympathoexcitation (17,31). Fukuda et al. (32) have suggested that the decrease in HR could be due to a direct effect of $\mathrm{CO}_{2}$ on cardiac pacemaker cells, but is not due to the baroreflex response. In contrast, Walker and Brizzee (33) have sug- gested that the baroreflex response plays an important role in the bradycardic response to hypercapnia. This discrepancy may be due to different levels of hypoxia, among other factors.

As stated above, renal denervation affects the fall in MAP and HR induced by the obstruction of the airways, demonstrating that renal nerves participate in these hemodynamic parameters. It is possible that the hypoxia-induced reflex increases the RSNA and consequently the renin overflow and norepinephrine spillover, including the vascular effect of these hormones, could increase the vascular resistance and reduce the MAP fall in S rats. Following renal denervation, the release of these hormones could be reduced, with this reduction contributing to an exacerbated fall of MAP in D rats. This point of view is supported by the findings of Bao et al. (34) showing that the circulating epinephrine (adrenal) may be an important regulator of arterial pressure in the setting of chronic episodes of hypoxia. Also, after acute renal denervation, Evans et al. (35) observed a reduction in renal plasma renin activity overflow and norepinephrine spillover in rabbits.

MAP falls during systemic hypoxia in the rat could be due to a fall in renal perfusion pressure and may influence renal function directly (36) or indirectly by stimulating the release of renin and generation of angiotensin II (37). However, in the present study, the fall of MAP during OA was observed in $S$ animals with intact renal nerve activity and without changes in sodium excretion or urine volume. These parameters were increased only in renal-denervated animals, despite an exacerbated fall of MAP. These data demonstrate that OA produces diuresis and natriuresis only after removal of the renal nerve, and is not dependent on the hypoxia-induced fall in MAP, as observed in our study.

Many studies have demonstrated that patients with obstructive sleep apnea have an abnormal nocturnal level of some vasoactive hormones $(6,8-10)$, such as atrial natriuretic peptide (ANP). The rise of plasma ANP concentration could result from increased transmural pressure (and thus atrial stretching) resulting from the obstructioninduced decrease in intrathoracic pressure at the peak of inspiration (38) and from the increased right atrial transmural pressure resulting from hypoxia-induced pulmonary vasoconstriction $(39,40)$.

Krieger (8) showed that apneic patients have nighttime increases in urine volume and its sodium content. These patients also exhibited increased urinary excretion of cyclic guanosine monophosphate, an intracellular messenger that mediates ANP actions, as well as increased plasma levels of ANP. This suggests that the respiratory efforts due to airway obstruction may influence the secretion of 
ANP and that this hormone may play an important role in the regulation of sodium urinary excretion during OA. An increase in urinary cyclic guanosine monophosphate concentration was also observed in rats with enhanced plasma ANP during OA. According to this study, 30 min after the recovery from $O A$, the urinary excretion volume and sodium content were still elevated when compared to basal values (4). These data are consistent with our study showing that the renal excretory parameters of renaldenervated rats remained elevated during the recovery period, which could be attributed to a direct effect of ANP on renal function.

\section{References}

1. Chiang AA. Obstructive sleep apnea and chronic intermittent hypoxia: a review. Chin J Physiol 2006; 49: 234-243.

2. Somers VK, Dyken ME, Clary MP, Abboud FM. Sympathetic neural mechanisms in obstructive sleep apnea. J Clin Invest 1995; 96: 1897-1904.

3. O'Donnell CP, Schwartz AR, Smith PL, Robotham JL, Fitzgerald RS, Shirahata M. Reflex stimulation of renal sympathetic nerve activity and blood pressure in response to apnea. Am J Respir Crit Care Med 1996; 154: 1763-1770.

4. Yalkut D, Lee LY, Grider J, Jorgensen M, Jackson B, Ott C. Mechanism of atrial natriuretic peptide release with increased inspiratory resistance. J Lab Clin Med 1996; 128: 322-328.

5. Berger AJ, Mitchell RA, Severinghaus JW. Regulation of respiration (first of three parts). N Engl J Med 1977; 297: 9297.

6. Carpagnano GE, Kharitonov SA, Resta O, FoschinoBarbaro MP, Gramiccioni E, Barnes PJ. Increased 8-isoprostane and interleukin- 6 in breath condensate of obstructive sleep apnea patients. Chest 2002; 122: 1162-1167.

7. Dibona GF, Kopp UC. Neural control of renal function. Physiol Rev 1997; 77: 75-197.

8. Krieger J. Hormonal control of sodium and water excretion in obstructive sleep apnoea. Scand J Urol Nephrol Suppl 1995; 173: 65-68.

9. Fletcher EC, Bao G, Li R. Renin activity and blood pressure in response to chronic episodic hypoxia. Hypertension 1999; 34: 309-314.

10. Svatikova A, Shamsuzzaman AS, Wolk R, Phillips BG, Olson LJ, Somers VK. Plasma brain natriuretic peptide in obstructive sleep apnea. Am J Cardiol 2004; 94: 529-532.

11. Veelken R, Sawin LL, Dibona GF. Dissociation of renal nerve and excretory responses to volume expansion in prehypertensive Dahl salt-sensitive and Dahl salt-resistant rats. Hypertension 1989; 13: 822-827.

12. Anderson RJ, Pluss RG, Berns AS, Jackson JT, Arnold PE, Schrier RW, et al. Mechanism of effect of hypoxia on renal water excretion. J Clin Invest 1978; 62: 769-777.

13. Nakamura KT, Ayres NA, Gomez RA, Robillard JE. Renal responses to hypoxemia during renin-angiotensin system
The data reported here obtained in anesthetized acute animals demonstrate a substantial participation of the RSNA in the control of renal function, HR and MAP during severe OA events in rats. We speculate that RSNA during $\mathrm{OA}$ is probably modulated by the balance of chemo- and cardiopulmonary reflexes since it is known that these reflexes are stimulated by OA episodes. Renal denervation-induced elevation of urine volume and sodium excretion suggests that the increase of the RSNA during OA does prevent the elevation of renal excretory function in $S$ animals.

inhibition in fetal lambs. Am J Physiol 1985; 249: R116R124.

14. Behm R, Mewes H, Muinck Keizer WH, Unger T, Rettig R. Cardiovascular and renal effects of hypoxia in conscious carotid body-denervated rats. J Appl Physiol 1993; 74: 27952800.

15. Malpas SC, Shweta A, Anderson WP, Head GA. Functional response to graded increases in renal nerve activity during hypoxia in conscious rabbits. Am J Physiol 1996; 271: R1489-R1499.

16. Honig A. Peripheral arterial chemoreceptors and reflex control of sodium and water homeostasis. Am J Physiol 1989; 257: R1282-R1302.

17. Rose CE Jr, Althaus JA, Kaiser DL, Miller ED, Carey RM. Acute hypoxemia and hypercapnia: increase in plasma catecholamines in conscious dogs. Am J Physiol 1983; 245: H924-H929.

18. Walker BR. Diuretic response to acute hypoxia in the conscious dog. Am J Physiol 1982; 243: F440-F446.

19. Koller EA, Buhrer A, Felder L, Schopen M, Vallotton MB. Altitude diuresis: endocrine and renal responses to acute hypoxia of acclimatized and non-acclimatized subjects. Eur J Appl Physiol Occup Physiol 1991; 62: 228-234.

20. Ramirez G, Hammond M, Agosti SJ, Bittle PA, Dietz JR, Colice GL. Effects of hypoxemia at sea level and high altitude on sodium excretion and hormonal levels. Aviat Space Environ Med 1992; 63: 891-898.

21. Colice G, Yen S, Ramirez G, Dietz J, Ou LC. Acute hypoxiainduced diuresis in rats. Aviat Space Environ Med 1991; 62: 551-554.

22. Dibona GF, Sawin LL. Renal nerves in renal adaptation to dietary sodium restriction. Am J Physiol 1983; 245: F322F328.

23. Souza DR, Mill JG, Cabral AM. Chronic experimental myocardial infarction produces antinatriuresis by a renal nervedependent mechanism. Braz J Med Biol Res 2004; 37: 285293.

24. Uggere TA, Abreu GR, Sampaio KN, Cabral AM, Bissoli NS. The cardiopulmonary reflexes of spontaneously hypertensive rats are normalized after regression of left ventricular 
hypertrophy and hypertension. Braz J Med Biol Res 2000; 33: 589-594.

25. McNicholas WT. Sleep apnoea. J Ir Coll Phys Surg 2008; 19: $53-56$

26. Dibona GF. Neural control of the kidney: functionally specific renal sympathetic nerve fibers. Am J Physiol Regul Integr Comp Physiol 2000; 279: R1517-R1524.

27. Shiomi T, Guilleminault C, Stoohs R, Schnittger I. Leftward shift of the interventricular septum and pulsus paradoxus in obstructive sleep apnea syndrome. Chest 1991; 100: 894902.

28. Levinson PD, Millman RP. Causes and consequences of blood pressure alterations in obstructive sleep apnea. Arch Intern Med 1991; 151: 455-462.

29. Oparil S, Chen SJ, Meng QC, Elton TS, Yano M, Chen YF. Endothelin-A receptor antagonist prevents acute hypoxiainduced pulmonary hypertension in the rat. Am J Physiol 1995; 268: L95-L100.

30. Denton KM, Shweta A, Anderson WP. Preglomerular and postglomerular resistance responses to different levels of sympathetic activation by hypoxia. J Am Soc Nephrol 2002; 13: 27-34

31. Bao G, Randhawa PM, Fletcher EC. Acute blood pressure elevation during repetitive hypocapnic and eucapnic hypoxia in rats. $J$ Appl Physiol 1997; 82: 1071-1078.

32. Fukuda Y, Sato A, Suzuki A, Trzebski A. Autonomic nerve and cardiovascular responses to changing blood oxygen and carbon dioxide levels in the rat. J Auton Nerv Syst 1989; 28: 61-74.
33. Walker BR, Brizzee BL. Cardiovascular responses to hypoxia and hypercapnia in barodenervated rats. J Appl Physiol 1990; 68: 678-686.

34. Bao G, Metreveli N, Li R, Taylor A, Fletcher EC. Blood pressure response to chronic episodic hypoxia: role of the sympathetic nervous system. J Appl Physiol 1997; 83: 95101.

35. Evans RG, Burke SL, Lambert GW, Head GA. Renal responses to acute reflex activation of renal sympathetic nerve activity and renal denervation in secondary hypertension. Am J Physiol Regul Integr Comp Physiol 2007; 293: R1247R1256.

36. Firth JD, Raine AE, Ledingham JG. The mechanism of pressure natriuresis. J Hypertens 1990; 8: 97-103.

37. Johns EJ. Role of angiotensin II and the sympathetic nervous system in the control of renal function. $J$ Hypertens 1989; 7: 695-701.

38. Amyot R, Michoud MC, Leduc T, Marleau S, Ong H, DuSouich $\mathrm{P}$, et al. Release of atrial natriuretic factor (ANF) induced by acute airway obstruction. Biochem Biophys Res Commun 1989; 160: 808-812.

39. Baertschi AJ, Teague WG. Alveolar hypoxia is a powerful stimulus for ANF release in conscious lambs. Am J Physiol 1989; 256: H990-H998.

40. Kawashima A, Kubo K, Hirai K, Yoshikawa S, Matsuzawa $\mathrm{Y}$, Kobayashi T. Plasma levels of atrial natriuretic peptide under acute hypoxia in normal subjects. Respir Physiol 1989; 76: 79-91. 